\title{
IMPORTÂNCIA DAS ANOTAÇOZS DOS CUIDADOS DE ENFERMAGEM
}

\author{
* Alaide Bittencourt Duarte \\ * Inácia Edésia M. dos Reis \\ * Vania Oliveira Santos
}

RBEn/06

DUARTE, A.B., REIS, I.E.M. e SANTOS V.O - Importância das anotaçōes dos cuidados de enfermagem. - Rev. Bras. Enf.; DF, $29: 83-91,1976$.

\section{1 - INTRODUÇAO}

$O$ atendimento ao paciente em Serviços de Saúde resulta para a instituição, em termos de registro, numa gama de informaçōes, que uma vez reunidas irāo constituir o prontuário do paciente.

Cada informaçāo anotada, indica uma Ação, que certamente foi desencadeada em razão direta de um problema apresentado pelo paciente, e do tratamento a esse dispensado.

Isto posto, situemos as Açōes de enfermagem nessa constelação de dados que uma vez tomada a forma de prontuário, passa a indicador da assistência prestada ao paciente.

Sendo a enfermagem, parte integrante dessa assistência, a anotaçāo dos cuidados prestados ao paciente, em função do mesmo, exprimirá toda a ação de enfermagem, desde sua concepção até a execuçāo.

De início pensamos em enfocar o problema da importância da anotação dos cuidados de enfermagem prestados, objetivando estudar um sistema de anotaçāo que tivesse a significância de indicador, no sentido de informar o que foi determinado em termos de cuidado de enfermagem para atender ao paciente, de controlador, no sentido de comprovar sua execução, e finalmente, de avaliar a qualidade do cuidado prestado, não apenas em termos do procedimento em si, mas desse em relação direta a reação do paciente ao cuidado recebido e da observação de enfermagem sobre o cuidado prestado.

Partimos da premissa que, se a "importância" da anotaçāo é um aspecto indiscutivel do problema, certamente, de uma forma ou de outra encontraríamos na prática material para, trabalhando sobre este, propor o sistema desejado.

Feitos os primeiros contatos, com vistas à aquisição de subsídios para o estudo, sentimos que o problema maior não estava na forma desordenada de anotar, ou mesmo na questão do que e de quem

* Docentes da Escola de Enfermagem da UERJ. 
DUARTE, A.B., REIS, I.E.M. e SANTOS V.O - Importância das anotaçōes dos cuidados de enfermagem. - Rev. Bras. Enf.; DF, $29: 83-91,1976$.

anotar, sim, na ausência de anotaçōes ou na hibridez da formulação daquelas encontradas.

Considerado o fato, percebemos a necessidade de reformular a proposição inicial.

Após reflexão, concluímos que, antes mesmo de qualquer proposição em termos de criação de sistema, tornar-se-ia nāo somente necessário, mas altamente importante, conhecer no momento atual a opinião do enfermeiro sobre a importância da anotação do cuidade de enfermagem prestado.

A luz dessa diagnose, num segundo momento poderíamos desenvolver um estudo onde nosso interesse maior seria o próprio mecanismo da anotação no contexto de um Sistema de Registro de Enfermagem.

Estávamos desejando experimentar um esquema terapêutico, não para tratar uma sindrome, mas um sinal apenas.

Colocamos anteriormente, que é indiscutivel a "importância da anotação dos cuidados prestados".

A premissa é, e será sempre verdadeira.

As razōes e fatores que determinaram a reformulação do trabalho não foram vistos pelo grupo, com alarde ou subjetiva preocupação.

Como enfermeiras, assumimos a parcela de responsabilidade que nos cabe no problema, que não é meramente institucional como parece a muitos, sim, de determinação de uma conduta profissional.

Reconhecido que a anotação de cuidados de enfermagem indica a prestação desses, e a reação do paciente aos mesmos, segundo a observação da enfermagem fica configurada sua condição de MEIO que dispomos para controlar e avaliar a assistência prestada.

Assim pois, estruturamos o estudo de modo a desenvolver-se em dois momentos; numa segunda etapa faremos uma pesquisa sobre as anotaçōes dos cuidados.
Trazemos hoje para os colegas, o produto da refiexão do grupo sobre o assunto e o resultado do levantamento da opiniāo dos enfermeiros sobre: anotação de cuidados de enfermagem e importância da anotação dos cuidados de enfermagem prestados.

\section{2 - CONSIDERAÇŌES GERAIS}

E sabido que vivemos um momento altamente questionável.

Procuramos um estabelecer de diretrizes que expresse decisivamente a ação de enfermagem.

Buscamos uma autonomia que certamente, mesmo considerando os tradicionais óbices de caráter institucional, carecemos provavelmente apenas, de um "assumir" de que a prestação da assistência de enfermagem somente se fará plena de autonomia, quando a ela for condicionado de forma irrestrita o planejar, o prescrever de cuidados e a anotação desses quando prestados.

Dissemos "assumir", no sentido de sentir e consentir, participar fazendo tudo aquilo que se fizer necessário para assegurar à profissāo seu pleno desenvolvimento.

Colocar a importância da anotação dos cuidados de enfermagem prestados como parte de um todo relativo a Sistema de Registro de Enfermagem diz bem da preocupação, que deve ser de todos nós, em dimensionar e definir com a devida praticidade as novas perspectivas da profissão.

Assumida a posição de que, toda assistência prevê planejamento e que a estrutura básica do plano de cuidado é a prescrição, concluímos, que, prescrever, executar e anotar é a própria natureza das açōes de enfermagem.

Para analisarmos o problema da importância de anotação dos cuidados de enfermagem prestados torna-se necessário situá-lo sob dois aspectos. 
DUARTE, A.B., REIS, I.E.M. e SANTOS V.O - Importáncia das anotaçסes dos cuidados de enfermagem. - Rev. Brus. Fof.; DF, 29 : 83-91, 1976.

Primeiramente em seu próprio contexto, ou seja, ligado intrinsecamente a metodologia da assistência de enfermagem.

Secundando essa colocaçāo, vamos encontrá-lo parte integrante do elenco de informaçōes que uma vez registradas vão constituir o prontuário do paciente.

As informaçōes registradas nesse, sāo cumulativas. Representando as açōes de todos os profissionais, envolvidos na assistência transforma-o em instrumento de singular importância para controle e avaliação dessa assistência prestada.

Isto posto, questionamos agora, especificamente, "cuidado de enfermagem" em seu aspecto conceitual.

E objeto de nossa atenção, paciente, familia e comunidade.

Operacionalizamos essa atenção, no momento em que identificamos problemas e definimos a ação de enfermagem que resultará no atendimento a uma situação criada em decorrência do problema existente.

A manifestação de um problema de ordem somática nos conduz a um tipo de atenção que projeta-se no paciente visando a enticiade mórbida.

Sabomos que a essa juntam-se manifestações que irāo determinar a individualidade do problema.

A primeira condição, ou seja a somática, é atendida em princípio em função de sintomás. Pressupẽe-se daí a indicação de uma terapêutica clínica ou cirúrgica e, em decorrência dessa, a prestação de um cuidado pela enfermagem.

E de todo importante enfatizar mais uma vez que vemos esse cuidado como parte integrante de um plano.

$A$ condição de cuidado planejado e executado pela enfermagem em conseqüência de uma indicação terapêutica, clínica ou círúrgica nos coloca em singular posiçāc.

Qua! seja: a de assumir responsabilidade de operacionalizar a prestação de um cuidado, indicado por outro elemento da equipe de saúde.

Planejamos e executamos esse cuidado espaldados nos princípios básicos de cuidados de enfermagem.

Se o fato é verdadeiro para os cuidados prescritos pelo enfermeiro para atender a problemas de enfermagem, não o é menos por tratar-se de cuidado decorrente de prescriçōes de outros profissionais.

Estamos a afirmar, que a enfermagem toma decisões por si mesma para resolver problemas relacionados com a atençäo do paciente.

Essas decisōes são tomadas a partir de conhecimentos científicos e técnicos, conhecimentos esses, que irāo resguardar a autonomia dessas decisões.

Tais afirmativas são premissas a qualquer situação de enfermagem.

Se assim não for, correremos o risco de continuarmos simplesmente executando tarefas para atender prescriçōes terapêuticas ou a simples normas institucionais.

Da determinação desta realidade, resulta a precisa posição da enfermagem face a prestação de cuidados decorrentes de prescriçōes terapêuticas e daqueles prestados pela enfermagem para atender ao paciente no que tange a problemas de enfermagem.

Configuramos a anotação do cuidado prestado como um meio que devemos dispor, para controlar e avaliar a assistência prestada.

Enfocamos o problema sob dois aspectos distintos.

Em qualquer uma das situações abordadas patenteia-se nossa responsabilidade e independência de ação no momento em que planejamos, prescrevemos, executamos ou delegamos a execuçāo do cuidado a outro membro da equipe e, finalmente, quando anotamos ou orientamos a anotação do cuidado prestado.

Segundo Horta, cuidado de enfermagem representa um conjunto ordenado 
DUARTE, A.B., REIS, I.E.M. e SANTOS V.O - Importância das anotaçōes dos cuidados de enfermagem. - Rev. Bras. Enf.; DF, $29: 83-91,1976$.

de açōes resultante de nossa percepção do comportamento do individuo, família ou comunidade. (9)

Esse conjunto ordenado de ação somente estará completo como unidade valorativa do plano de cuidado, quando assumirmos de fato, a condição de que a prestação de um cuidado exige incondicionalmente uma anotação.

Vimos que o prontuário do paciente é constituído de informaçōes indicadoras da participação dos profissionais envolvidos no processo da assistência.

Ora, nāo há o que discutir quanto a nossa participação nesse processo.

Há o que lamentar. Lamentar o fato de nós mesmos, estarmos permitindo, pela falta de anotação ou pela qualidade de algumas, que não conste da documentação do paciente a unidade indicadora de nossas açōes.

\section{3 - MATERIAL E MÉTODO}

\section{1 - Campo de Pesquisa}

Não objetivamos essencialmente estudar o problema centrando-o na área de trabalho do enfermeiro ou seja, uma análise em função de sua unidade específica de atividades.

Era importante detectar sua opiniāo, como enfermeiro, qualquer que fosse sua área de atuação.

Entretanto tornou-se necessário situálo como amostra, em um espaço físico que pelo seu porte de trabalho e finalidade constitui-se numa amostragem de alta significação.

Assim, buscamos opiniāo de enfermeiros cujas atividades se desenvolviam em hospitais universitários, de previdência e ensino e de assistência médico social.

\section{2 - Metodologia}

Considerando que nosso objetivo era identificar, no momento atual, a opiniāo do enfermeiro sobre "anotação de cuida- dos de enfermagem" e, importância da anotaçāo dos cuidados prestados, elabıramos um formulário que permitisse ao colega expressar sua conceituação sobre o assunto e indicar à luz das declaraçōes apresentadas «ua opiniāo sobre a "importância" do mesmo.

\section{3 - Conceituaçāo de Termos}

AÇAO - Tudo que se faz; modo de atuar.

ANOTAÇAO - Ato de anotar; acontamento; nota.

ASSISTENCIA - Ato de assistir; prestar auxílio; amparo; atenção; auxílio técnico.

ASSUMIR - Tomar sobre si; encarregar-se de; entrar em exercício de.

ATENÇAO - Vigilância, cuidado. Aplicação da inteligência, e dos sentidos, para a compreensão.

ATIVIDADE - Qualidade de ativo; faculdade de operar.

ATUAÇAO - Ato de atuar; forma de exercer ação e de agir.

AVALIAÇAO - Ato ou efeito de avaliar; apreciação; valor determinado por peritos.

AVALIAR - Determinar o valor ou a valia de: apreciar o merecimento de.

CONCEITO - Idéia; opiniāo; juizo.

CONDUTA - Ato de conduzir; procedimento; comportamento.

COLABORAR - Trabalhar juntamente com outro ou outros; cooperar.

CUIDADO - Ato de atender ou prestar serviços; desvelo; incumbência; encargo.

DOCUMENTO - Declaração escrita para servir de prova; testemunho; confirmação.

EVOLUÇÃO - Desenvolvimento gradual e progressivo de uma idéia. Desenvolvimento de um ser, órgão, sistema, processo mórbido ou teoria.

HOSPITAL UNIVERSITARIO - Hospital integrante de uma universidade. 
DUARTE, A.B., REIS, I.E.M. e SANTOS V.O - Importância das anotaçōes dos cuidados de enfermagem. - Rev. Bras. Ene.; DF, 29 : 83-91, 1976.

HOSPITAL DE ENSINO - Hospital que mesmo não pertencendo à universidade, serve de campo de ensino.

HOSPITAL DE PREVIDENCIA - Além da assistência previdenciária promove 0 ensino.

IMPORTANCIA - Qualidade de importante; valor.

MÉTODo - Processo racional para chegar a determinado fim; maneira de proceder; processo ou técnica para chegar ao conhecimento ou demonstração da verdade.

NECESSIDADE - Aquilo que é absosutamente necessário; falta do que é necessário.

PARTICIPAR - Ter ou tomar parte em alguma coisa; comunicar-se; entrar em contato.

PLANEJAMENTO - Ato de planejar; fazer um plano; programar.

PLANO - Projeto, intento, programa.

PRESCRIÇAO - Ato ou efeito de prescrever; ordem terminantemente expressa; ditame; preceito; formulário. Dispor em papel os cuidados a serem prestados ao paciente.

PROCEDIMENTO - Ato ou efeito de proceder; comportamento; conduta.

QUALIDADE - Aquilo que caracteriza uma coisa; propriedade; modo de ser; espécie.

REGISTRAR - Escrever ou lançar no registro; consignar por escrito.
REGISTRO - Ato ou efeito de registrar.

RESPONSABIIIDADE - Qualidade do que é responsável; obrigaçāo de responder por certos atos; próprios ou de outrem.

SISTEMA - Conjunto de partes coordenadas entre si; combinação de partes, de modo que concorram para certo resultado; método; processo.

\section{RESULTADOS E COMENTARIOS}

A aplicação de 100 formulários resultou na formulaçāo de $91(91 \%)$ conceitos, uma vez que $5(5 \%)$ formulários não expressaram conceituação e 4 (4\%) conceitos foram considerados evasivos.

Estudados os conceitos, esses foram grupados segundo a idéia central em 5 unidades básicas a saber: Idéia básica - incidência.

1. Documentação escrita no prontuário $-24(24 \%)$.

2. Fornece dados para pesquisas $6(6 \%)$.

3. Instrumento utilizado para orientar a equipe $-30(30 \%)$.

4. Instrumento utilizado para acompanhar a evolução clínica - $33(33 \%)$.

5. Instrumento utilizado para avaliar a assistência $-35(35 \%)$.

A opiniāo do enfermeiro sobre a importância da anotação dos cuidados apresentados foi expressa à luz das 6 declaraçōes apresentadas o que resultou:

\begin{tabular}{ccccc}
\hline Declaração & Confirmaram & $\begin{array}{c}\text { Não } \\
\text { Confirmaram }\end{array}$ & Discordaram & $\begin{array}{c}\text { Não } \\
\text { Responderam }\end{array}$ \\
\hline 1 & $82(82 \%)$ & $4(4 \%)$ & $7(7 \%)$ & $7(7 \%)$ \\
2 & $89(89 \%)$ & $2(2 \%)$ & $2(2 \%)$ & $7(7 \%)$ \\
3 & $11(11 \%)$ & $7(7 \%)$ & $67(67 \%)$ & $15(15 \%)$ \\
4 & $70(70 \%)$ & $10(10 \%)$ & $15(15 \%)$ & $5(5 \%)$ \\
5 & $84(84 \%)$ & $10(10 \%)$ & $2(2 \%)$ & $4(4 \%)$ \\
6 & $4(4 \%)$ & $9(9 \%)$ & $71(71 \%)$ & $16(16 \%)$ \\
\hline
\end{tabular}


DUARTE, A.B., REIS, I.E.M. e SANTOS V.O - Importância das anotaçōes dos cuidados de enfermagem. - Rev. Bras. Enf.; DF, $29: 83-91,1976$.

\section{Comentários:}

Sobre a forma redacional dos conceitos expressos é válido (mesmo sem dados estatísticos) comentar que os mesmos demonstraram seu uso se não inccrreto, nejo menos pouco adequado de uma linguagem técnica.

Pela formulação dos conceitos, detectamos idéias básicas, mas essas não enfocavam precisamente 0 aspecto da assistência de enfermagem.

Quanto a importância da anotação dos cuidados prestados foi considerado importante como indicador, e que estas devem ser acompanhadas de informaçōes relativas an paciente.

Entretanto $11 \%$ não considerou necessário anotar sistematicamente os cuidados prestados. $15 \%$ discordaram que a anotação permite avaliar a assistência e $4 \%$ achou que não é necessário anotar por existirem outros registros.

\section{CONCLUSOES E RECOMENDAÇOES}

Os dados analisados nos permitiram concluir que é altamente significativo a defasagem ainda existente entre o preconizado no atual dimensionamento da enfermagem e o que encontramos no exercício da profissão.

O fato nos permite propor:

1 - Que os serviços de enfermagem desenvolvam programas para atender a necessidade de atualização e treinamento do grupo.

2 - Que os programas sejam elaborados e desenvolvidos partindo de um estabelecer de prioridades do grupo.

3 - Que as estratégias acionadas permitam um ensino que atenda as peculiaridades do Treinando.

4 - Que as escolas participem decisivamente dessa atualização através integração docente-assistencial.

\section{REFERENCIA BIBLIOGRAFICA}

01. BROWN, Esther Lucile - Nuevas Dimensiones en el cuidado de los pacientes - México, La Prensa Médica Mexicana, 1971.

02. Carvalho, A.C., - Plano de Cuidados de Enfermagem como uma das funçōes do Enfermeiro-Chefe. Revista da Escola de Enfermagem da U.S.P. - 1:108-117, março, 1968.

03. CaRVALHO, A.C. - Consideraçōes sobre o Ensino de Campo na Enfermagem: Revista Brasileira de Enfermagem - 5:149-153, out./dez., 1972

04. CarValho, v. - Dos Princípios e Proposiçōes da Observação Sistematizada na Enfermagem - Revista Brasileira de Enfermagem - 5:30-45 julho/setembro, 1971.

05. DOURADO, H.G. - Funções do Enfermeiro - Revista Brasileira de Enfermagem - 1, 2, 3: Editorial, junho, 1968.

06. FUERST, Elinor V. \& WOLFF, Lu Verne - Principios Fundamentales de Enfermeria - 5 ed., México, La Prensa Médica Mexicana, 1972.

07. HENDERSON, Virginia - Principios Básicos sobre cuidados de Enfermagem. Rio de Janeiro, Editora Hélios, 1962.

08. HARMER, Bertha \& HENDERSON, Virginia - Tratado de Enfermeria Teórica y Practica. 2. ed., México, La Prensa Médica Mexicana. 1970.

09. HORTA, W. - A observação sistematizada na identificação dos problemas de enfermagem em seus aspectos físicos - Revista Brasileira de Enfermagem - 2: 214-219, abril/junho 1974.

10. KOZIER, Barbara Blackwood \& DU GAS, Beverly Witter - Tratado de Enfermeria Practica. México, Editorial Interamericana, 1974.

11. KRON, Thora - Manual de Enfermeria. México, Nueva Editorial Interamericana, 1973. 
DUARTE, A.B., REIS, I.E.M. e SANTOS V.O - Importáncia das anotaçōes dos cuidados de enfermagem. - Rev. Bras. Enf.; DF, 29 : 83-91, 1976.

12. Mc CLAIN, Esther \& GRAGG, Shirley Hawke - Principios Cientificos da Enfermagem. Rio de Janeiro, Editora Cientifica, 1970.

13. MAC GREGOR, Frances Cooke - Las ciencias sociales y la Enfermeria. México, La Prensa Médica Mexicana, 1968.

14. NORDMARK, Madelyn Titus \& ROHWEDER, Anne W. - Principios Cientificos Aplicados a la Enfermeria. México, La Prensa Médica Mexicana, 1972.

15. PAIM, Lygia et alii - Iniciamento à metodologia do Processo de Enfermagom. Rio de Janiero, ABen, 1974.
16. PEREZ, Maria del Carmen Ledesma Introduccion a la Enfermeria. México Editorial Limusa, 1974.

17. PRICE, Alice - Tratado de Enfermeria - México, Editorial Interamericana, 198.9.

18. RIBEIRO, C.M. e GARCIA, T.L. Padrōes mínimos para campo de prática de estudantes de enfermagem - Revista Brasileira de Enfermagem - 5: 372-374, outubro, 1968.

19. RIBEIRO, C.M. - Auditoria de Serviços de Enfermagem - Revista Brasileira de Enfermagem - 4: 91 103 julho/setembro, 1972.

20. SOUZA, Elvira de Felice et alii - Novo Manual de Enfermagem 5. ed., Rio de Janeiro, Bruno Buccini, 1972. 
DUARTE, A.B., REIS, I.E.M. e SANTOS V.O - Importância das anotações dos cuidados de enfermagem. - Rev. Bras. Enf.; DF, $29: 83-91,1976$.

CONCEITO DOS ENFERMEIROS SOBRE ANOTAÇÓES DE CUIDADOS DE ENFERMAGEM

\begin{tabular}{|c|c|c|c|}
\hline $\begin{array}{l}\text { N.O DE } \\
\text { ORDEM }\end{array}$ & IDEIA CENTRAL & N. ${ }^{\circ}$ & $\%$ \\
\hline 1. & $\begin{array}{l}\text { Documentação escrita dos registros, } \\
\text { contidos no prontuário do paciente. }\end{array}$ & 24 & 24 \\
\hline 2. & Fornecer dados para pesquisas. & 6 & 6 \\
\hline 3. & $\begin{array}{l}\text { Instrumento utilizado para orientar a } \\
\text { equipe. }\end{array}$ & 30 & 30 \\
\hline 4. & $\begin{array}{l}\text { Instrumento utilizado para acompa- } \\
\text { nhar a evolução clínica do paciente. }\end{array}$ & 33 & 33 \\
\hline 5. & $\begin{array}{l}\text { Instrumento utilizado para avaliar a } \\
\text { assistência. }\end{array}$ & 35 & 35 \\
\hline
\end{tabular}

* DOS CEM FORMULÁRIOS APLICADOS, CINCO NAO EMITIRAM CONCEITOS E QUATRO RESPONDERAM DE FORMA EVASIVA. 


\begin{tabular}{|c|c|c|c|c|c|c|c|}
\hline \multirow{2}{*}{ 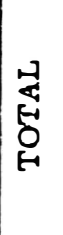 } & 80 & 음 & 옴 & ఏ & હ્ન & ఫ & 음 \\
\hline & $\stackrel{\circ}{z}$ & ○ & ○ & ఫ్రి & ఫ & 임 & ఫ్ન \\
\hline \multirow{2}{*}{ 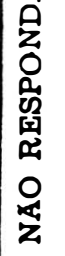 } & sீ & $\sigma$ & $\kappa$ & $\stackrel{2}{2}$ & $n$ & + & $\stackrel{\oplus}{-}$ \\
\hline & $\stackrel{\circ}{z}$ & $\sigma$ & $\tau$ & 2 & م" & + & $\stackrel{\oplus}{\sim}$ \\
\hline \multirow{2}{*}{ 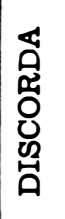 } & ઠீ & $\sigma$ & N & 5 & $\stackrel{2}{-1}$ & N & $\approx$ \\
\hline & $\stackrel{\circ}{z}$ & $\sigma$ & N & 5 & $\stackrel{2}{\sim}$ & N & $\approx$ \\
\hline \multirow{2}{*}{ 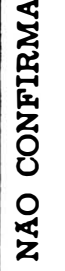 } & s。 & + & $\boldsymbol{N}$ & $\tau$ & 우 & 이 & $\infty$ \\
\hline & $\stackrel{\circ}{z}$ & $*$ & N & $\sigma$ & 이 & 어 & o) \\
\hline \multirow{2}{*}{ 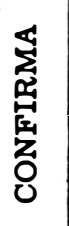 } & $\delta 8$ & జ & § & $\Rightarrow$ & ㅇ & め゙ & + \\
\hline & $\stackrel{\circ}{\ddot{z}}$ & జ & ஜ & $\Rightarrow$ & 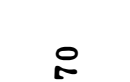 & $\varpi$ & + \\
\hline \multicolumn{2}{|c|}{ 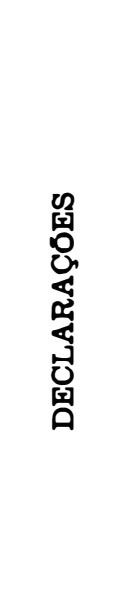 } & 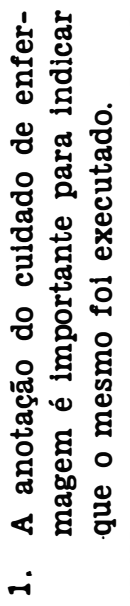 & 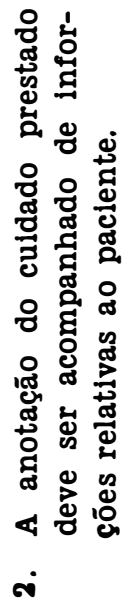 & 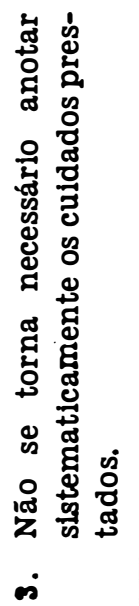 & 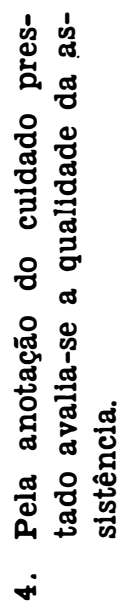 & 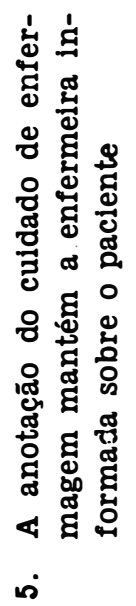 & 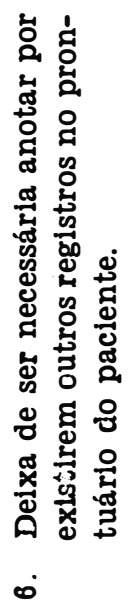 \\
\hline
\end{tabular}

